\title{
Association analysis between the tag single nucleotide polymorphisms of DENND1A and the risk of polycystic ovary syndrome in Chinese Han women
}

Ya-nan Zhu ${ }^{1,2+}$, Yi-ting Zhang ${ }^{2+}$, Qin Liu², Shan-mei Shen ${ }^{3}$, Xiang Zou ${ }^{4}$, Yun-xia Cao ${ }^{5}$, Wen-jun Wang ${ }^{6}$, Long Yi ${ }^{2}$, Qian Gao ${ }^{2}$, Wei-dong Yang ${ }^{1 *}$ and Yong Wang ${ }^{2^{*}}$ (D)

\begin{abstract}
Background: The DENND1A gene is one of the most important sites associated with polycystic ovary syndrome (PCOS). We attempted to analyze the correlation between five single nucleotide polymorphisms (SNPS) in the DENNDIA gene and the development of PCOS.

Methods: A total of 346 PCOS patients and 225 normal ovulatory women were involved in the case-control study. Clinical variables and hormones were recorded. According to the Hap Map database, five tagging SNPs (rs2479106, rs2768819, rs2670139, rs2536951 and rs2479102) in the DENND1A gene were identified. The TaqMan probe and the PCR-RFLP (restriction fragment length polymorphism) methods were used for revealing these genotypes. TaqMan Genotype Software was used to analyze the alleles of the five SNPs.

Results: Linkage disequilibrium and the gene frequency analysis demonstrated that the CCGGG haplotype might increase the risk of PCOS $(P=0.038, O R=1.89,95 \% \mathrm{Cl}=1.027-3.481)$. Significant differences were found in genotypic and allelic distributions at the rs2536951 and rs2479102 loci between PCOS women and controls $(P<0.001)$. The LH levels and LH/FSH ratios were higher in PCOS patients than in the control group. A detailed analysis revealed that for the rs2479106 locus, these two values were significantly different in the control subjects who had AA, AG and GG genotypes ( $P=0.013$ and $P=0.007$, respectively), and for the rs2468819 locus, these two values were significantly different among the PCOS patients with AA, AG and GG genotypes $(P=0.013$ and 0.002 , respectively).

Conclusions: The tagging SNPs rs2479106 and rs2468819 in the DENND1A gene are associated with PCOS in the Chinese population, whereas rs2670139, rs2536951 and rs2479102 are not correlated with PCOS in the same population.
\end{abstract}

Keywords: Polycystic ovary syndrome, Genetic polymorphism, DENND1A

\footnotetext{
* Correspondence: ywdong2001@sohu.com; yongwang@nju.edu.cn

†Ya-nan Zhu and Yi-ting Zhang contributed equally to this work.

${ }^{1}$ Department of Endodontics, Nanjing Stomatological Hospital, Medical

School of Nanjing University, Nanjing, Jiangsu, China

${ }^{2}$ State Key Laboratory of Analytical Chemistry for Life Science \& Jiangsu Key

Laboratory of Molecular Medicine, Medical School, Nanjing University,

Nanjing, Jiangsu, China

Full list of author information is available at the end of the article
}

(c) The Author(s). 2020 Open Access This article is distributed under the terms of the Creative Commons Attribution 4.0 International License (http://creativecommons.org/licenses/by/4.0/), which permits unrestricted use, distribution, and reproduction in any medium, provided you give appropriate credit to the original author(s) and the source, provide a link to the Creative Commons license, and indicate if changes were made. The Creative Commons Public Domain Dedication waiver (http://creativecommons.org/publicdomain/zero/1.0/) applies to the data made available in this article, unless otherwise stated. 


\section{Background}

Polycystic ovary syndrome (PCOS) is a highly complex gynecological endocrine disease affecting up to $10 \%$ of women of reproductive age [1]. It is the most common endocrine disorder in the gynecological and endocrinological clinic in China, where $50 \%$ of patients suffer from PCOS $[2,3]$. The clinical manifestations of PCOS can affect many organs, including the hypothalamus, pituitary, ovary, adrenal gland, and pancreas, leading to a higher degree of genetic heterogeneity. The loose ovulation and endocrine disorder are the main reasons for female infertility, which severely compromises women's physical and mental health. Furthermore, PCOS may be accompanied by an increased risk of diabetes mellitus, glucose intolerance, hypertension, atherogenic dyslipidemia, non-alcoholic fatty liver disease, systemic inflammation and coagulation disorders. The etiology of PCOS has not been clearly defined. It is believed that genetic factors may play important roles in its pathogenesis, and at least 70 candidate responsible genes have been identified [4]. A milestone event for the first attempt of a genome-wide association study (GWAS) on PCOS focused on chromosomes 2p16.3, 2p21 and 9q33.3 in Han Chinese women. The corresponding gene loci were LHCGR (luteinizing hormone/choriogonadotropin receptor), THADA (thyroid associated protein) and DENNDIA (DENN/MADD domain-containing 1A) [5].

Two years later, another GWAS project suggested eight new candidate risk loci for the development of PCOS in the Chinese population. These loci, including follicle stimulating hormone receptor (FSHR), C9orf3, YAP1, RAB5B, HMGA2, TOX3, insulin receptor (INSR), and SUMO1P1 [6], are found to be associated with PCOS etiology by being involved in the synthesis of reproductive hormones, functional regulation of gonadotropin and insulin resistance [7]. Another large GWAS was recently published focusing on European subjects [8]. The strongest associations in Europeans are found to be in DENND1A and THADA loci, and additional associations have also been revealed at loci containing LHCGR, RAB5/SUOX, FSHR and YAP1 [9]. These studies prompted us to search for more loci that might account for the origin and pathophysiology of PCOS. Various genetic polymorphisms have been described for PCOS [10], and the association of single nucleotide polymorphisms (SNPs) with the occurrence and development of PCOS has been confirmed. Three SNPs (rs13405728 in LHCGR gene; rs13429458 in THADA gene and rs2479106 in DENND1A gene) have been identified to be genetic variants of PCOS by GWAS in Han Chinese populations [11, 12] .

Among those SNP loci that may have an impact on PCOS, the DENND1A gene, one of the DENND family members, has attracted substantial attention. DENND1A is one of the causal factors expressed in the theca follicle [13]. DENND1A SNPs are associated with metabolic disturbances and endocrine disorders [14]. They also have a profound impact on the establishment of hyperandrogenic PCOS phenotypes [15]. DENND1A, which encodes the protein connecdenn 1 , consists of 22 exons and extends over 500,000 bases. DENND1A does not facilitate endocytosis and receptor-mediated turnover. Connecdenn 1, one of the proteins encoded by the DENND1A gene, facilitates these functions in the lipid bilayer. It has a clathrin-binding domain that is localized in the $\mathrm{N}$-terminus of the protein and is associated with the metabolism of phosphoinsitol-3-phosphates and other lipids [16]. $D E N N D 1 A$ is important in facilitating endocytosis and receptor-mediated turnover $[17,18]$. DENN domain proteins form a new class of membrane transporters which regulate Rab GTPases [16]. On the other hand, DENND1A-encoded domain binds ERAP1 (endoplasmic reticulum amino acid peptidase 1 ) as a negative regulator. ERAP1 expression is elevated in the serum of obese PCOS patients [19]. We speculated that DENND1A might affect the pathogenesis of PCOS through dysregulation of ERAP1 in those PCOS patients with a risk allele of DENND1A.

The DENND1A gene is associated with PCOS in both Han Chinese and European women, although the associations are focused on different SNPs [20-22]. Some SNPs in the DENND1A gene, including rs10818854, rs2479106 and rs10986105, are reported as susceptible loci $[12,14]$. Among the many polymorphisms of DENND1A genes, rs2479106 and rs10818854 polymorphisms have received much attention. Several studies have previously suggested that the rs2479106 and rs10818854 polymorphisms are associated with an increased risk of PCOS [23, 24].

In addition to the rs2479106 and rs10818854 polymorphisms, other SNPs related to the physical signs and disease characteristics of PCOS might also exist. In this study, The LD pattern of the DENND1A gene including almost all the SNPs is presented in a full picture based on the Hap Map Phase III Han Phase database in NCBI by Hap lo View software version 4.2. Based on the LD pattern, we attempted to select a few SNPs (with linkage and representation) to determine whether polymorphisms of the DENND1A gene were related to PCOS, both at the individual SNP and haplotype levels, in the Han Chinese population. As most studies suggest that the polymorphism site rs2479106 in the DENND1A gene is related to PCOS susceptibility, we chose the other four SNPs which have a strong relation with rs2479106. Finally, five tagging SNPs including rs2479106, rs2768819, rs2670139, rs2536951 and rs2479102 were selected as the representative loci for investigating the association between the tag SNPs of DENND1A and the risk of PCOS in Chinese Han women. 


\section{Methods}

\section{Subjects}

Using the 2003 Rotterdam Criteria (The Rotterdam ESHRE/ASRM-Sponsored PCOS Consensus Workshop Group, 2004), we recruited 346 PCOS patients and 225 women without PCOS, both being the Chinese Han women. The samples were collected from subjects recruited from Drum Tower Hospital in Nanjing, Department of Obstetrics and Gynecology, Anhui Medical University in Hefei, and Memorial Hospital of Sun YatSen University, Guangzhou in China. Patients with PCOS were diagnosed according to the 2003 Rotterdam Criteria (The Rotterdam ESHRE/ASRM-Sponsored PCOS Consensus Workshop Group, 2004). The Rotterdam Criteria require at least two of the following indicators for diagnosis of PCOS: (1) Oligo- and/or anovulation; (2) Clinical and/or biochemical signs of hyperandrogenism; and (3) Polycystic ovaries and exclusion of other aetiologies (congenital adrenal hyperplasias, androgen-secreting tumors, Cushing's syndrome).

The women in the control group came to visit the clinic for other reasons (such as tubal factor infertility or their husbands' infertility). Some of them had given birth to one child or more. Their menstrual cycles were normal (<32 days) and exclusion criteria were hirsutism, insulin resistance, other property of hyperandrogenism and obesity.

The study was approved by the Medical School of Nanjing University. The patients and the control women provided informed consent and volunteered to participate in the study.

\section{Clinical and biochemical determination}

First, clinical variables were recorded, including height and body weight. Body mass index (BMI) was calculated as weight $(\mathrm{kg})$ divided by height $(\mathrm{m})$. Second, we collected the peripheral blood samples between 8 am and 9 am after a 12-h overnight fast. For those women in the menstrual cycle, blood samples were collected from the 3 rd day to the 5th day. For those women who had amenorrhea, blood samples were collected at a random day. A series of biochemical measurements were carried out to obtain the hormone levels, including luteinizing hormone $(\mathrm{LH})$, total testosterone $(\mathrm{T})$, follicle-stimulating hormone (FSH) and estradiol (E2) using radioimmunoassay (Beijing North Institute of Biological Technology, China).

\section{Characterization of linkage disequilibrium (LD) and selection of tag SNPs}

The DENND1A gene is located on chromosome 9q33.3. The SNPs genotyped were tagging SNPs in the DENND1A gene based on the HapMap database (www. hapmap.org, HapMap database release no. R2/phase III, population: $\mathrm{CHB}$ ) for the Chinese Han population (Additional file 1: Figure S1-1, S1-2). The site rs2479106 and the other four SNPs were selected with strong linkage in Block 12. The pair-wise correlations between rs2479106 and these SNPs were the same (measured as D' $=95 \%$ ). Finally, the five tagging SNPs, rs2479106, rs2768819, rs2670139, rs2536951 and rs2479102, were selected for the following association study. (Fig. 1).

\section{Genotyping for polymorphisms}

Genomic DNA was extracted from blood samples with an SBS UltraPure ${ }^{\mathrm{rm}}$ Genome DNA kit (SBS Genentech, Shanghai, China) and examined by a Thermo Scientific Nano Drop ${ }^{\text {тм }} 2000$ spectrophotometer (Thermo Electron, Massachusetts, USA). Genomic DNA was stored at $20 \mathrm{ng} / \mu \mathrm{l}$ in ultrapure water and at $-80^{\circ} \mathrm{C}$ until further genotyping analysis for polymorphisms by the TaqMan SNP genotyping assay. PCR amplification was conducted in a total volume of $10 \mu \mathrm{l}$ composed of $5 \mu \mathrm{l} 2 \mathrm{X}$ TaqMan Master Mix, $0.5 \mu \mathrm{l} 20 \mathrm{X}$ Assay Working Stock, $1 \mu \mathrm{l}$ sample Genomic DNA (20 ng per well), and $3.5 \mu \mathrm{l}$ nuclease-free water by the One Step One Plus RT-PCR System (Applied Biosystems). PCR was carried out as follows: $95^{\circ} \mathrm{C}$ Ampli Taq Gold UP Enzyme Activation for $10 \mathrm{~min}$, followed by 40 cycles consisting of $95^{\circ} \mathrm{C}$ denaturation for $15 \mathrm{~s}$ and $60^{\circ} \mathrm{C}$ annealing/extension for $1 \mathrm{~min}$, ending with a single extension of $15 \mathrm{~min}$ at $60^{\circ} \mathrm{C}$.

\section{Statistical analysis}

SPSS 17.0 was used for statistical analyses. $P<0.05$ was considered statistically significant. The age, AAM, BMI and serum hormone level data were presented as the means \pm SD. The differences in hormone levels of different genotypes in the two groups were detected by oneway analysis of variance (ANOVA). The genotype data were calculated with the expectation-maximization (EM) algorithm, presented in Haplo View version 4.2, and analyzed by the SHEsis online software (http://analysis.bio-x. $\mathrm{cn}$ /myAnalysis.php). Next, it was measured with the pairwise LD analysis. The alleles of the five SNPs were analyzed using TaqMan Genotype Software. The $\chi^{2}$ test of the $2 \times 3$ tables was used to compare the genotypic distributions and Hardy-Weinberg distribution of genotypes between patients and controls. The differences among the three genotypes were presented by the Turkey test.

\section{Results}

Characterization of the clinical features of PCOS and the controls

Characterization of the clinical and biochemical features of the PCOS patients $(n=346)$ and control women $(n=$ 225) are listed in Table 1 . PCOS patients were diagnosed under the Rotterdam Criteria. Significant differences in BMI, E2, LH and LH/FSH ratios were revealed between the two groups. Compared with the controls, PCOS 


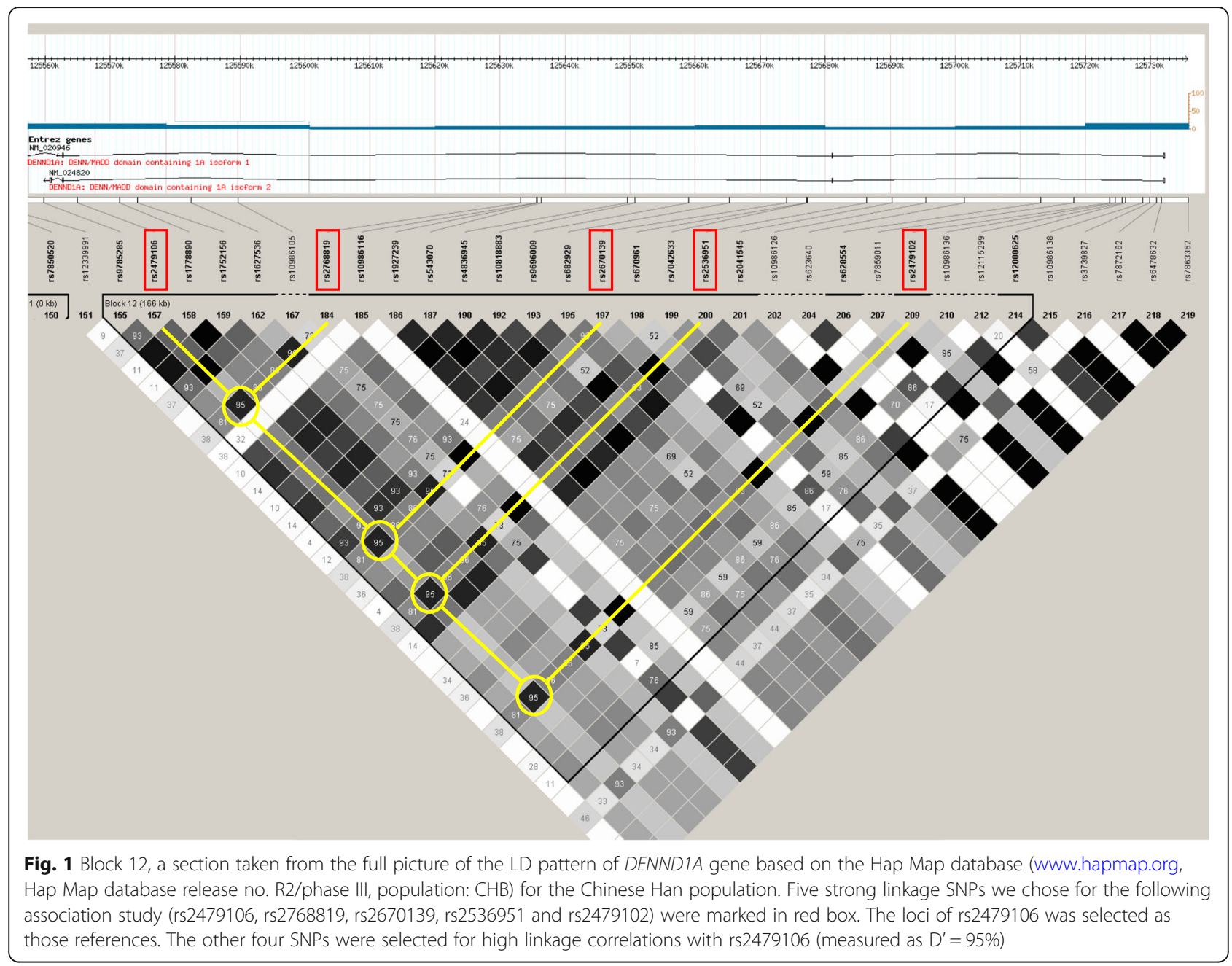

patients were characterized by higher BMI, E2, LH and $\mathrm{LH} / \mathrm{FSH}$ ratios.

\section{The LD structure and haplotype-based association analyses}

Based on the Hap Map Phase III database (release no. $\mathrm{R}^{2}$ / phase III, population: $\mathrm{CHB}$ ), five SNPs were identified in the promoter of the $D E N N D 1 A$ gene $(\mathrm{MAF}>0.05)$. Five tag SNPs were revealed by Haplo View, through LD analysis and gene frequency analysis according to NCBI (Fig. 2a). The pairwise correlation of two SNPs was indicated as the value in the diamond (measured as D'). The Hap lo View of
LD pattern from the data of our research were presented in Fig. 2b (measured as D') and Fig. 2c (measured as $R^{2}$ ). On the one hand, these data showed that the 5 SNPs had linkage correlation within each other. On the other hand, these SNPs also have their distinct functions.

LD structures with the genotype data for SNPs were reexamined to obtain haplotype-based association analyses. There was a significant difference of haplotype frequencies in the CCGGG haplotype between the patients and the controls $(P=0.0386, \quad \mathrm{OR}=1.891,95 \% \mathrm{CI}=1.027-3.481)$ (Table 2). The CCGGG haplotype might enhance susceptibility to PCOS.

Table 1 Clinical characteristics of PCOS and control subjects

\begin{tabular}{|c|c|c|c|c|c|c|c|c|}
\hline Groups & $\mathrm{N}$ & $\begin{array}{l}\text { BMl* } \\
(\mathrm{kg} / \mathrm{m} 2)\end{array}$ & $\begin{array}{l}\text { E2* } \\
\text { (pMol/L) }\end{array}$ & $\begin{array}{l}\mathrm{T} \\
\text { (nMol/L) }\end{array}$ & $\begin{array}{l}\mathrm{LH}^{*} \\
(\mathrm{IU} / \mathrm{L})\end{array}$ & $\begin{array}{l}\text { FSH } \\
(\mathrm{IU} / \mathrm{L})\end{array}$ & $\mathrm{LH} / \mathrm{FSH}^{*}$ & PRL(IU/L) \\
\hline PCOS & 346 & $\begin{array}{l}29.69 \pm \\
3.14\end{array}$ & $102.19 \pm 20.3$ & $\begin{array}{l}2.84 \pm \\
0.15\end{array}$ & $\begin{array}{l}9.79 \pm \\
1.76\end{array}$ & $\begin{array}{l}6.78 \pm \\
0.52\end{array}$ & $1.44 \pm 0.03$ & $5.75 \pm 1.06$ \\
\hline Control & 225 & $\begin{array}{l}22.03 \pm \\
2.35\end{array}$ & $60.19 \pm 10.91$ & $\begin{array}{l}2.26 \pm \\
0.32\end{array}$ & $\begin{array}{l}5.22 \pm \\
0.91\end{array}$ & $\begin{array}{l}7.87 \pm \\
1.06\end{array}$ & $0.70 \pm 0.12$ & $5.96 \pm 1.03$ \\
\hline
\end{tabular}

${ }^{*} P<0.05$ between the PCOS and control groups. Data are expressed as mean \pm standard deviation. $B M I$ body mass index, E2 estradiol, $T$ testosterone, $L H$ luteinizing hormone, FSH follicle-stimulating hormone, $P R L$ prolactin 


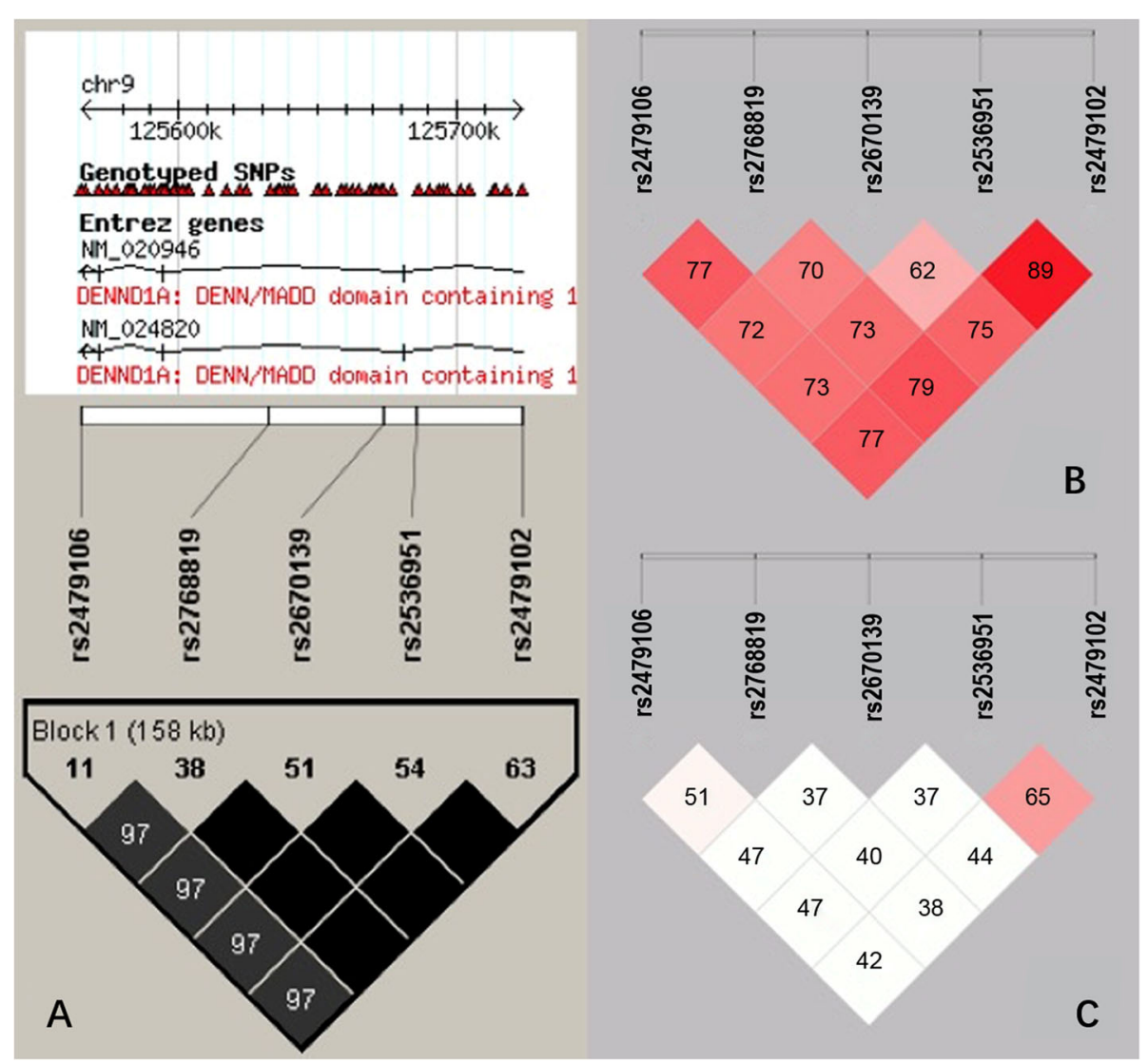

Fig. 2 The LD analysis pattern of the five strong linkage SNPs (rs2479106, rs2768819, rs2670139, rs2536951 and rs2479102) selected from the DENNDIA gene. $\mathbf{a}$ is the pattern according to database of NCBI for Chinese Han population. It is spanning from $2 \mathrm{~kb}$ upstream to $5 \mathrm{~kb}$ downstream of DENND1A within 125,600 k to125700k. The value within the diamond represents the pair-wise correlation between SNPs (measured as $D^{\prime}$ ). The Hap lo View of LD pattern from the data of our research were presented in (b) (measured as $\left.D^{\prime}\right)$ and $(\mathbf{c})\left(\right.$ measured as $\left.R^{2}\right)$

\section{Allele distribution and SNP genotype analysis}

Distributions of allelic and genotypic frequencies of five SNPs (rs2479106, rs2768819, rs2670139, rs2536951 and rs2479102) are listed in Tables 3 and 4. The distributions of the rs2479106 genotype were 0.3380 for AG, 0.6130 for AA, and 0.0490 for GG. The data of the rs2768819 genotype were 0.3488 for AG, 0.6192 for AA and 0.3198 for GG. The distributions of the rs 2670139 genotype were 0.2826 for CT, 0.0917 for CC and 0.6257 for TT. Analyses of rs2536951 resulted in 0.2962 for AG, 0.6044 for AA and 0.0994 for GG. For rs2479102, data analysis pointed to 0.2882 for CT, 0.0961 for CC and 0.6157 for TT. The genotypic distribution did not

Table 2 Correlation between the haplotypes containing the 5 tag SNPs including rs2479106, rs2768819, rs2670139, rs2536951 and rs2479102 and the PCOS risk in cases and controls

\begin{tabular}{lllll}
\hline & Case (freq) & Control (freq) & Pearson's $P$ & Odds Ratio [95\%Cl] \\
\hline CCGGG & $19(0.201)$ & $39(0.112)$ & $0.038^{*}$ & $1.89[1.027 \sim 3.481]$ \\
TTAAA & $64(0.680)$ & $212(0.602)$ & 0.399 & $1.26[0.733 \sim 2.176]$ \\
TTAGA & $3(0.032)$ & $4(0.012)$ & 0.207 & $2.55[0.567 \sim 11.498]$ \\
\hline
\end{tabular}

${ }^{*} P<0.05$ between the cases and controls reveal significant differences for rs2768819, rs2479106 or rs2670139 between PCOS and healthy women $(P=0.842$, 0.277 and 0.465 , for rs2768819, rs2479106 and rs2670139, respectively). No significant differences were found regarding allelic distribution between the two groups $(P=0.985$, 0.37 and 0.23 for rs2768819, rs2479106 and rs2670139, respectively). Interestingly, significant differences in the genotypic distribution for rs2536951 and rs2479102 between the patients and controls were identified $(P<0.001)$, and similar differences were also found in allelic distribution $(P<0.001$, Table 3$)$. The distributions of genotypes were in agreement with Hardy-Weinberg equilibrium.

Clinical and metabolic characteristics among the different genotypes

Clinical and metabolic characteristics among the different genotypes (AA, AG and GG) of the five SNPs (rs2479106, rs2768819, rs2670139, rs2536951 and rs2479102) in patients and the controls are listed in Table 4. For rs2479106, serum LH levels and the ratios of LH/FSH were significantly different among AA, AG, and GG genotypes in the controls $(P=0.013$ and $P=0.007)$, while there 
Table 3 Genotypes and alleles of rs2479106, rs2768819, rs2670139, rs2536951 and rs2479102 in PCOS cases and controls

\begin{tabular}{|c|c|c|c|c|c|c|c|}
\hline \multirow[t]{2}{*}{ rs2479106 } & \multicolumn{3}{|c|}{ Genotypes, n (\%) } & \multirow[t]{2}{*}{$p$-value ${ }^{a}$} & \multicolumn{2}{|c|}{ Alleles, n (\%) } & \multirow[t]{2}{*}{$p$-value } \\
\hline & $\overline{A G}$ & $\mathrm{AA}$ & GG & & $A$ & G & \\
\hline Control & $78(0.347)$ & $140(0.622)$ & $7(0.031)$ & 0.277 & $358(0.796)$ & $92(0.204)$ & 0.37 \\
\hline PCOS & $115(0.332)$ & $210(0.607)$ & $21(0.061)$ & & $535(0.773)$ & $157(0.227)$ & \\
\hline \multirow[t]{2}{*}{ rs2768819 } & \multicolumn{3}{|c|}{ Genotypes, n (\%) } & $p$-value ${ }^{a}$ & \multicolumn{2}{|c|}{ Alleles, n (\%) } & $p$-value \\
\hline & $A G$ & $\mathrm{AA}$ & GG & & A & G & \\
\hline Control & $31(0.365)$ & $52(0.612)$ & $2(0.024)$ & 0.842 & $135(0.794)$ & $35(0.206)$ & 0.985 \\
\hline PCOS & $89(0.344)$ & $161(0.622)$ & $9(0.035)$ & & $411(0.793)$ & $107(0.207)$ & \\
\hline \multirow[t]{2}{*}{ rs2670139 } & \multicolumn{3}{|c|}{ Genotypes, n (\%) } & $p$-value ${ }^{a}$ & \multicolumn{2}{|c|}{ Alleles, n (\%) } & $p$-value \\
\hline & $\mathrm{CT}$ & CC & $\pi$ & & $C$ & $\mathrm{~T}$ & \\
\hline Control & $65(0.284)$ & 25(0.109) & $139(0.607)$ & 0.465 & $115(0.251)$ & $343(0.749)$ & 0.23 \\
\hline PCOS & $89(0.282)$ & $25(0.079)$ & $202(0.639)$ & & $139(0.220)$ & 493(0.780) & \\
\hline \multirow[t]{2}{*}{ rs2536951 } & \multicolumn{3}{|c|}{ Genotypes, n (\%) } & $p$-value ${ }^{a}$ & \multicolumn{2}{|c|}{ Alleles, n (\%) } & $p$-value \\
\hline & $A G$ & $\mathrm{AA}$ & GG & & A & G & \\
\hline Control & $67(0.307)$ & $116(0.532)$ & $35(0.161)$ & $<0.001$ & $299(0.686)$ & $137(0.314)$ & $<0.001$ \\
\hline PCOS & $82(0.288)$ & 188(0.660) & 15(0.053) & & $458(0.804)$ & 112(0.196) & \\
\hline \multirow[t]{2}{*}{ rs2479102 } & \multicolumn{3}{|c|}{ Genotypes, n (\%) } & $p$-value ${ }^{a}$ & \multicolumn{2}{|l|}{ Alleles, n (\%) } & $p$-value \\
\hline & $\mathrm{CT}$ & CC & $\pi$ & & C & $\mathrm{T}$ & \\
\hline Control & $61(0.269)$ & $37(0.163)$ & $129(0.568)$ & $<0.001$ & $135(0.297)$ & $319(0.703)$ & $<0.001$ \\
\hline PCOS & $86(0.304)$ & $12(0.042)$ & $185(0.654)$ & & $110(0.194)$ & $456(0.806)$ & \\
\hline
\end{tabular}

$P$-value ${ }^{\mathrm{a}}$ is based on the genotype frequencies versus control.

$P$-value ${ }^{\mathrm{b}}$ is based on the allele frequencies versus control.

was no difference in the patients (Fig. 3a, b). LH concentrations and LH/FSH ratios of rs2468819 significantly differed among AA, AG, and GG genotypes in the patients $(P=0.013$ and 0.002 , respectively) (Fig. 3c, d). However, no impact on controls was found. The LH/FSH ratios of rs2536951 demonstrated a significant difference among AA, AG, and GG genotypes in the controls $(P=0.026)$, but there was no difference in PCOS patients. No significant differences were found among AA, AG, and GG genotypes of rs2670139 and rs2479102, in either PCOS patients or controls. There were no significant differences in BMI or levels of any other serum hormones, such as testosterone, prolactin, E2/T, LH, FSH, LH/FSH and estradiol, in the other two genotypes in PCOS patients and controls (Table 4).

\section{Discussion}

In this study, we provided data suggesting that the promoter polymorphism of the DENND1A gene could impact the incidence of PCOS, which is consistent with recent reports showing that the rs $2479106 \mathrm{G}$ allele is significantly associated with decreased sensitivity to PCOS [25] [26]. A GWAS analysis also resulted in a similar finding [5].

In PCOS women, LH, FSH, LH/FSH ratio and TSH were significantly increased compared with the control group [27]. Subsequent quantitative trait analysis revealed that there was an association between the rs2768819 GG genotype and increased levels of LH as well as a higher LH/FSH ratio in PCOS patents. For the control subjects, higher LH levels and increased LH/FSH ratios were observed in those with the rs2479106 AG genotype. However, additional samples are required to confirm the association at the other haplotype loci.

Evidence of familial aggregation and geographical impact on the clinical traits suggested that the pathogenesis of PCOS involves a variety of genetic and environmental factors. A conditional logistic regression analysis showed that both rs10818854 and rs2479106 are associated PCOS [5]. Another GWAS study demonstrated that the SNP site rs346803513 in DENND1A is correlated with the disease pathogenesis of PCOS [28]. However, for Chinese women belonging to the Hui ethnic group, rs13405728 is found to be associated with PCOS. In contrast, no statistically significant correlation with PCOS is found for rs13429458 and rs2479106 in this ethnic group [29]. Two of the five SNPs (rs10986105 and rs10818854) on 9q33.3 are suggested to be associated with PCOS in the Chinese population [5]. Although the risk variant of rs 2479106 has been successfully replicated in at least some of the Chinese populations, it is not found to be associated with PCOS in European cohorts [20]. Examination of the PCOS patients in this study indicates that the variant might be related to hyperandrogenism or irregular menses [20]. In European 
Table 4 Clinical and metabolic characteristics of genotypes containing rs2479106, rs2768819, rs2670139, rs2536951 and rs2479102 in PCOS cases and controls

\begin{tabular}{|c|c|c|c|c|c|c|c|c|}
\hline Genotypes & Control & & & $p$-value & PCOS & & & $p$-value \\
\hline rs2479106 & AG & $\mathrm{AA}$ & GG & & $A G$ & $\mathrm{AA}$ & GG & \\
\hline Age (years) & $33.53 \pm 0.65$ & $32.58 \pm 0.49$ & $34.40 \pm 2.27$ & 0.771 & $26.97 \pm 0.72$ & $26.15 \pm 0.60$ & $33.00 \pm 0.49$ & 0.227 \\
\hline BMI (kg/m2) & $22.72 \pm 0.49$ & $21.51 \pm 0.26$ & $23.41 \pm 0.62$ & 0.143 & $23.33 \pm 0.72$ & $23.06 \pm 0.54$ & $19.96 \pm 0.49$ & 0.268 \\
\hline $\mathrm{E} 2(\mathrm{pg} / \mathrm{ml})$ & $63.06 \pm 7.36$ & $57.13 \pm 4.40$ & $42.87 \pm 5.23$ & 0.436 & $209.61 \pm 25.42$ & $256.54 \pm 16.61$ & $102.19 \pm 28.39$ & 0.184 \\
\hline $\mathrm{T}(\mathrm{nm} / \mathrm{l})$ & $2.93 \pm 0.17$ & $2.04 \pm 0.32$ & $1.34 \pm 0.32$ & 0.147 & $3.99 \pm 1.06$ & $6.27 \pm 2.15$ & $2.84 \pm 1.05$ & 0.727 \\
\hline $\mathrm{E} 2 / \mathrm{T}(\mathrm{In})$ & $21.33 \pm 1.72$ & $72.21 \pm 25.10$ & $33.11 \pm 2.87$ & 0.335 & $92.12 \pm 12.49$ & $93.92 \pm 12.68$ & $35.98 \pm 5.49$ & 0.797 \\
\hline LH(IU/I) & $5.94 \pm 0.64$ & $4.79 \pm 0.29$ & $2.56 \pm 0.83$ & $0.013^{*}$ & $15.73 \pm 1.31$ & $17.11 \pm 1.19$ & $9.79 \pm 1.48$ & 0.318 \\
\hline FSH(IU/I) & $8.40 \pm 0.66$ & $7.90 \pm 0.31$ & $7.00 \pm 0.41$ & 0.701 & $7.17 \pm 0.50$ & $6.27 \pm 0.33$ & $6.78 \pm 0.29$ & 0.551 \\
\hline LH/FSH & $0.78 \pm 0.07$ & $0.64 \pm 0.04$ & $0.36 \pm 0.11$ & $0.007^{*}$ & $2.99 \pm 0.19$ & $2.94 \pm 0.20$ & $1.44 \pm 0.12$ & 0.068 \\
\hline $\operatorname{PRL}(\mu \mathrm{g} / \mathrm{l})$ & $4.67 \pm 1.35$ & $6.31 \pm 1.78$ & $1.79 \pm 0.03$ & 0.724 & $14.62 \pm 1.59$ & $18.21 \pm 2.10$ & $5.75 \pm 1.05$ & 0.365 \\
\hline \multirow[t]{2}{*}{ rs2768819 } & Control & & & $p$-value & PCOS & & & $p$-value \\
\hline & $A G$ & AA & GG & & $A G$ & AA & GG & \\
\hline Age (years) & $32.6 \pm 0.77$ & $33.58 \pm 0.51$ & $34.00 \pm 1.00$ & 0.935 & $28.63 \pm 0.84$ & $27.24 \pm 0.80$ & $22.5 \pm 0.65$ & 0.269 \\
\hline BMI (kg/m2) & $22.7 \pm 0.59$ & $21.93 \pm 0.32$ & $21.69 \pm 2.79$ & 0.465 & $13.52 \pm 0.88$ & $23.30 \pm 0.79$ & $19.72 \pm 0.86$ & 0.678 \\
\hline $\mathrm{E} 2(\mathrm{pg} / \mathrm{ml})$ & $62.7 \pm 8.65$ & $61.36 \pm 5.27$ & $56.36 \pm 25.64$ & 0.983 & $197.51 \pm 32.63$ & $192.59 \pm 17.67$ & $250.90 \pm 30.35$ & 0.899 \\
\hline $\mathrm{T}(\mathrm{nm} / \mathrm{l})$ & $2.71 \pm 0.33$ & $2.09 \pm 0.14$ & $1.67 \pm 0.65$ & 0.339 & $2.01 \pm 0.15$ & $2.55 \pm 0.26$ & $3.35 \pm 0.64$ & 0.225 \\
\hline $\mathrm{E} 2 / \mathrm{T}(\mathrm{In})$ & $20.5 \pm 3.37$ & $72.51 \pm 10.75$ & $32.75 \pm 2.51$ & 0.241 & $109.08 \pm 16.66$ & $75.45 \pm 15.55$ & $84.77 \pm 17.94$ & 0.917 \\
\hline LH(IU/I) & $5.45 \pm 0.52$ & $5.19 \pm 0.34$ & $4.58 \pm 1.41$ & 0.905 & $11.75 \pm 1.26$ & $16.66 \pm 1.61$ & $31.70 \pm 2.34$ & $0.013^{*}$ \\
\hline FSH(IU/I) & $6.59 \pm 0.28$ & $8.04 \pm 0.38$ & $8.42 \pm 0.44$ & 0.057 & $6.58 \pm 0.54$ & $7.06 \pm 0.50$ & $6.21 \pm 0.54$ & 0.779 \\
\hline LH/FSH & $0.89 \pm 0.09$ & $0.68 \pm 0.04$ & $0.54 \pm 0.14$ & 0.222 & $1.85 \pm 0.16$ & $2.44 \pm 0.21$ & $5.26 \pm 0.69$ & $0.002^{*}$ \\
\hline $\operatorname{PRL}(\mu \mathrm{g} / \mathrm{l})$ & $4.58 \pm 2.91$ & $7.64 \pm 2.18$ & $1.80 \pm 0.06$ & 0.583 & $15.16 \pm 1.69$ & $19.53 \pm 3.71$ & $24.43 \pm 1.33$ & 0.618 \\
\hline \multirow[t]{2}{*}{ rs 2536951} & Control & & & $p$-value & PCOS & & & $p$-value \\
\hline & AG & $\mathrm{AA}$ & GG & & $A G$ & AA & GG & \\
\hline Age (years) & $33.3 \pm 0.99$ & $32.61 \pm 0.52$ & $32.30 \pm 0.96$ & 0.667 & $27.43 \pm 0.68$ & $25.98 \pm 0.62$ & $30.00 \pm 2.00$ & 0.166 \\
\hline BMI (kg/m2) & $22.3 \pm 0.61$ & $21.71 \pm 0.31$ & $21.44 \pm 0.80$ & 0.442 & $23.41 \pm 0.79$ & $22.99 \pm 0.58$ & $26.89 \pm 3.16$ & 0.413 \\
\hline $\mathrm{E} 2(\mathrm{pg} / \mathrm{ml})$ & $61.0 \pm 6.03$ & $56.40 \pm 4.51$ & $59.45 \pm 12.17$ & 0.861 & $217.63 \pm 29.31$ & $247.00 \pm 19.28$ & $232.68 \pm 80.20$ & 0.699 \\
\hline $\mathrm{T}(\mathrm{nm} / \mathrm{l})$ & $2.85 \pm 0.37$ & $2.28 \pm 0.36$ & $1.09 \pm 0.07$ & 0.268 & $3.43 \pm 0.83$ & $4.01 \pm 0.48$ & $2.12 \pm 0.56$ & 0.67 \\
\hline $\mathrm{E} 2 / \mathrm{T}(\mathrm{In})$ & $20.7 \pm 9.80$ & $70.70 \pm 27.74$ & $28.84 \pm 1.41$ & 0.412 & $93.06 \pm 14.93$ & $89.39 \pm 12.39$ & $107.24 \pm 9.50$ & 0.947 \\
\hline LH(IU/I) & $6.19 \pm 0.51$ & $5.07 \pm 0.38$ & $5.25 \pm 0.70$ & 0.284 & $15.74 \pm 1.47$ & $17.21 \pm 1.37$ & $12.58 \pm 2.02$ & 0.739 \\
\hline $\mathrm{FSH}(\mathrm{IU} / \mathrm{l})$ & $8.10 \pm 0.59$ & $8.14 \pm 0.37$ & $7.70 \pm 0.79$ & 0.93 & $6.77 \pm 0.51$ & $6.36 \pm 0.38$ & $7.36 \pm 0.71$ & 0.65 \\
\hline LH/FSH & $0.88 \pm 0.07$ & $0.64 \pm 0.04$ & $0.72 \pm 0.08$ & $0.026^{*}$ & $2.35 \pm 0.17$ & $2.91 \pm 0.21$ & $1.75 \pm 0.44$ & 0.139 \\
\hline $\mathrm{PRL}(\mu \mathrm{g} / \mathrm{l})$ & $1.73 \pm 0.69$ & $5.09 \pm 1.85$ & $3.79 \pm 0.63$ & 0.98 & $13.15 \pm 1.55$ & $18.21 \pm 2.36$ & $18.33 \pm 8.65$ & 0.358 \\
\hline \multirow[t]{2}{*}{ rs2479102 } & Control & & & $p$-value & PCOS & & & $p$-value \\
\hline & $\mathrm{CT}$ & $\mathrm{CC}$ & $\pi$ & & $C T$ & $C C$ & $\pi$ & \\
\hline Age (years) & $33.2 \pm 0.71$ & $33.40 \pm 1.06$ & $32.80 \pm 0.50$ & 0.561 & $27.56 \pm 0.74$ & $23.33 \pm 2.60$ & $26.60 \pm 0.63$ & 0.218 \\
\hline BMI (kg/m2) & $22.3 \pm 0.55$ & $22.94 \pm 1.05$ & $21.64 \pm 0.26$ & 0.197 & $23.40 \pm 0.90$ & $20.25 \pm 1.88$ & $23.48 \pm 0.61$ & 0.443 \\
\hline E2(pg/ml) & $62.4 \pm 7.30$ & $47.53 \pm 4.73$ & $59.70 \pm 4.79$ & 0.525 & $190.30 \pm 26.79$ & $215.11 \pm 31.41$ & $240.79 \pm 20.58$ & 0.35 \\
\hline $\mathrm{T}(\mathrm{nm} / \mathrm{l})$ & $2.75 \pm 0.33$ & $1.20 \pm 0.10$ & $2.16 \pm 0.29$ & 0.191 & $3.24 \pm 0.89$ & $2.48 \pm 0.52$ & $3.36 \pm 0.45$ & 0.915 \\
\hline $\mathrm{E} 2 / \mathrm{T}(\mathrm{In})$ & $21.9 \pm 3.88$ & $24.52 \pm 3.66$ & $64.40 \pm 21.94$ & 0.374 & $84.89 \pm 12.45$ & $89.30 \pm 7.27$ & $103.02 \pm 14.29$ & 0.702 \\
\hline LH(IU/I) & $5.68 \pm 0.58$ & $5.06 \pm 0.60$ & $5.04 \pm 0.35$ & 0.619 & $14.86 \pm 1.61$ & $21.22 \pm 5.02$ & $15.56 \pm 1.14$ & 0.424 \\
\hline $\mathrm{FSH}(\mathrm{IU} / \mathrm{I})$ & $7.90 \pm 0.61$ & $9.02 \pm 0.90$ & $7.86 \pm 0.34$ & 0.129 & $6.94 \pm 0.47$ & $6.06 \pm 0.30$ & $6.32 \pm 0.39$ & 0.594 \\
\hline LH/FSH & $0.80 \pm 0.07$ & $0.59 \pm 0.07$ & $0.67 \pm 0.04$ & 0.243 & $2.13 \pm 0.18$ & $3.55 \pm 0.91$ & $2.72 \pm 0.22$ & 0.092 \\
\hline PRL( $\mu g / l)$ & $4.64 \pm 3.35$ & $3.79 \pm 0.63$ & $5.76 \pm 1.64$ & 0.902 & $13.82 \pm 1.57$ & $22.26 \pm 7.07$ & $18.52 \pm 2.49$ & 0.38 \\
\hline
\end{tabular}


Table 4 Clinical and metabolic characteristics of genotypes containing rs2479106, rs2768819, rs2670139, rs2536951 and rs2479102 in PCOS cases and controls (Continued)

\begin{tabular}{|c|c|c|c|c|c|c|c|c|}
\hline \multirow[t]{2}{*}{ rs2670139 } & \multicolumn{3}{|l|}{ Control } & \multirow[t]{2}{*}{$p$-value } & \multicolumn{3}{|l|}{ PCOS } & \multirow[t]{2}{*}{$p$-value } \\
\hline & $C T$ & $C C$ & $\pi$ & & $\mathrm{CT}$ & $\mathrm{CC}$ & $\pi$ & \\
\hline Age (years) & $33.2 \pm 0.67$ & $32.79 \pm 1.11$ & $32.66 \pm 0.51$ & 0.807 & $27.59 \pm 0.71$ & $25.50 \pm 2.50$ & $26.12 \pm 0.60$ & 0.318 \\
\hline BMI (kg/m2) & $22.20 \pm 0.5$ & $22.33 \pm 1.17$ & $21.61 \pm 0.25$ & 0.458 & $23.40 \pm 0.83$ & $21.73 \pm 2.01$ & $23.20 \pm 0.55$ & 0.857 \\
\hline $\mathrm{E} 2(\mathrm{pg} / \mathrm{ml})$ & $57.9 \pm 6.98$ & $60.14 \pm 12.70$ & $55.72 \pm 3.41$ & 0.89 & $214.98 \pm 29.90$ & $201.58 \pm 49.11$ & $250.40 \pm 17.80$ & 0.536 \\
\hline $\mathrm{T}(\mathrm{nm} / \mathrm{l})$ & $2.81 \pm 0.31$ & $2.15 \pm 0.75$ & $2.01 \pm 0.26$ & 0.198 & $3.37 \pm 0.85$ & $2.46 \pm 0.90$ & $6.37 \pm 2.04$ & 0.592 \\
\hline $\mathrm{E} 2 / \mathrm{T}(\mathrm{In})$ & $22.0 \pm 3.89$ & $23.19 \pm 7.55$ & $66.16 \pm 23.76$ & 0.371 & $95.37 \pm 15.37$ & $86.28 \pm 11.45$ & $91.78 \pm 11.84$ & 0.979 \\
\hline LH(IU/I) & $5.39 \pm 1.00$ & $4.84 \pm 1.49$ & $5.03 \pm 0.35$ & 0.786 & $14.86 \pm 1.51$ & $22.83 \pm 8.24$ & $17.63 \pm 1.23$ & 0.945 \\
\hline $\mathrm{FSH}(\mathrm{IU} / \mathrm{I})$ & $7.65 \pm 1.10$ & $8.23 \pm 2.35$ & $8.09 \pm 0.36$ & 0.129 & $6.57 \pm 0.47$ & $6.27 \pm 0.38$ & $6.73 \pm 0.36$ & 0.272 \\
\hline $\mathrm{LH} / \mathrm{FSH}$ & $0.78 \pm 0.14$ & $0.63 \pm 0.16$ & $0.64 \pm 0.04$ & 0.476 & $2.29 \pm 0.19$ & $3.73 \pm 1.54$ & $2.83 \pm 0.20$ & 0.138 \\
\hline PRL( $\mu g / l)$ & $2.53 \pm 0.77$ & $1.79 \pm 0.04$ & $4.63 \pm 1.42$ & 0.769 & $12.82 \pm 1.37$ & $16.33 \pm 6.65$ & $18.17 \pm 2.16$ & 0.294 \\
\hline
\end{tabular}

${ }^{*} P<0.05$. Statistical analyses were carried out by analysis of covariance to correct for age and BMI. Data are expressed as mean \pm standard deviation. $B M I$ body mass index, E2 estradiol, $T$ testosterone, $L H$ luteinizing hormone, FSH follicle-stimulating hormone

patients, one mistyped SNP (rs189947178, A/C) that may alter the structural conformation of the DENND1A protein is more prevalent in PCOS patients with moderate hirsutism [26]. Among the Caucasians, the rs2479106 G allele is associated with a decreased susceptibility to PCOS [21]. In the Bahraini Arabic population, the DENND1A
SNPs including rs2479106, rs10986105 and rs10818854 are associated with PCOS [23]. It has been previously suggested that the association of rs10818854 and rs10986105 polymorphisms with PCOS is dependent on the ethnic origin of the population [12]. A meta-analysis showed that the DENND1A SNPs are associated with the pathogenesis

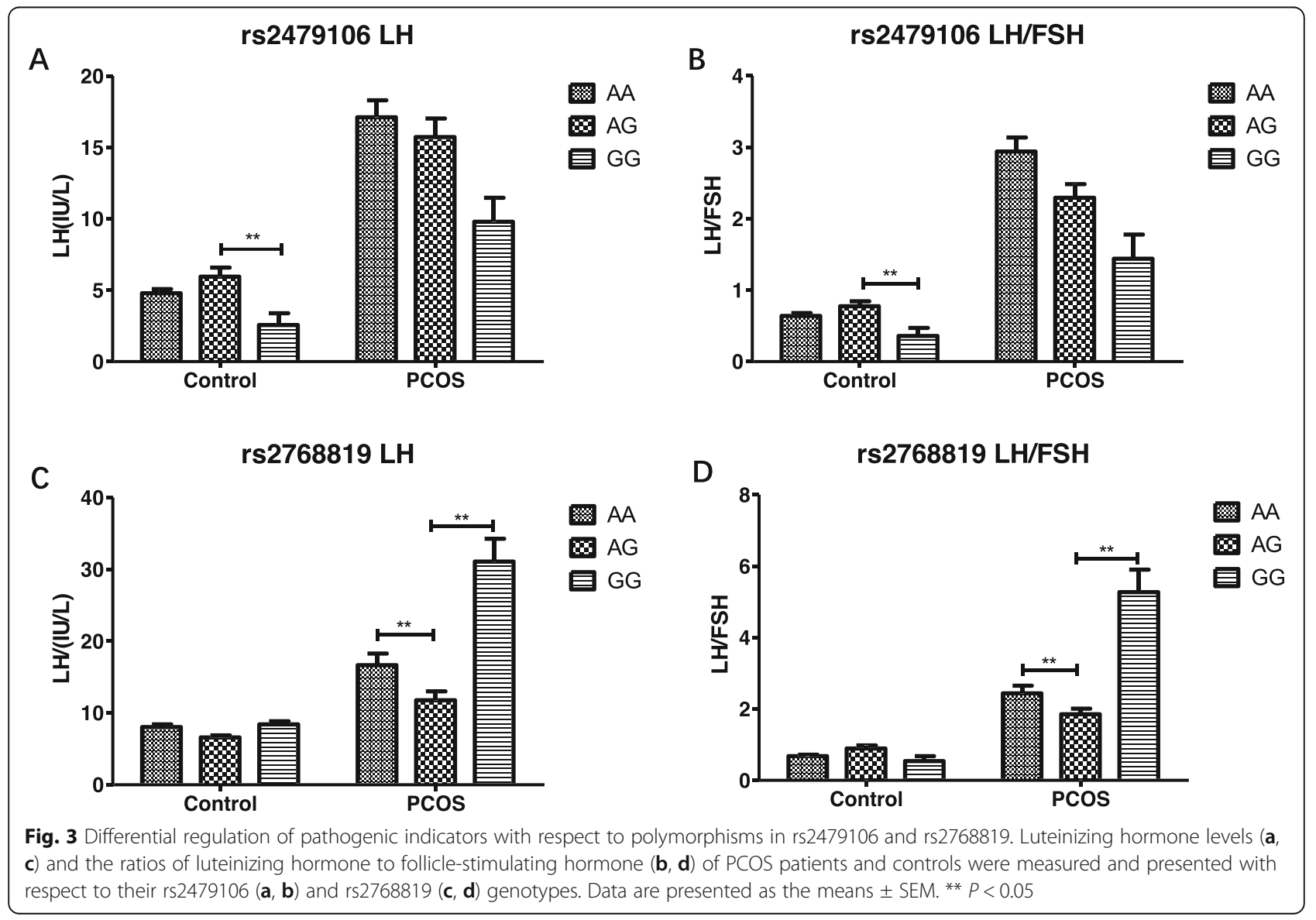


of PCOS [30]. Again, a strong ethnic influence has been suggested, as rs2479106, rs10818854, and rs10986105 are associated with PCOS only in Asian populations, whereas rs10818854 and rs10986105 are correlated with PCOS in Caucasians. No correlation has been established between rs2479106 and PCOS.

This study focused on the five SNPs associated with the DENND1A gene among subjects in Eastern China in a large sample study; the results were persuasive and credible, thus filling the gap on the research of this area. The correlation between the DENND1A gene-related SNPs and PCOS incidence is subject to the ethnicity of the population and environmental modulation; whether our findings can be applied to populations other than Chinese or even Chinese in other geographic areas remains uncertain.

In this study, significant differences in LH levels and LH/FSH ratios of PCOS patients and controls were revealed based on the rs2479106 and rs2768819 polymorphisms $(P<0.05)$. DENND1A, which plays an important role in the expression and metabolism of hormones, is associated with PCOS through the regulation of hyperandrogenemia [31]. On the other hand, the variation of DENND1A may affect the exocytosis of gonadotropins. $D E N N D 1 A$ is also associated with androgen secretion and expression. The DENND1A.V2 protein is increased in theca cells of PCOS patients [13]. Higher levels of DENND1A.V2 in theca cells can promote CYP17A1 and CYP11A1 gene transcription and androgen synthesis, leading to hyperandrogenemia of PCOS [13]. In several studies, the association between PCOS and SNPs of DENND1A gene has been demonstrated and replicated. Taken together, these data strongly support a contributory role of DENND1A in the development of PCOS. Furthermore, it can be speculated that the DENND1A gene has an impact on the increase of androgen and the release of gonadotropin in PCOS patients.

\section{Conclusions}

Our data provide further support for the established relationship between DENND1A polymorphisms and the pathogenesis of PCOS in a Chinese Han population from Eastern China. The tagging SNPs rs2479106 and rs2468819 in the DENND1A gene are associated with PCOS, whereas rs2670139, rs2536951 and rs2479102 are not correlated with PCOS in the same population. The sample size used in this study is not large enough, and may lead to limited power of the association test. We will continue collecting more samples and expect to have a larger sample size in future studies. Further research is required to fully reveal the mechanisms by which the tag SNPs in DENND1A may exert pathophysiological regulation in promoting or suppressing PCOS development. These observations and further clarifications may have both diagnostic and therapeutic implications for this disorder.

\section{Supplementary information}

Supplementary information accompanies this paper at https://doi.org/10. 1186/s12881-019-0945-1.

Additional file 1: Figure S1. A full picture of the LD pattern of DENND1A gene. The SNPs genotyped were tagging SNPS in DENND1A gene based on the Hap Map database (www.hapmap.org, Hap Map database release no. R2/phase III, population: CHB) for the Chinese Han population Figure S1-1. is the clear one, and the linkage association was marked in Figure S1-2. In Block 12, the pair-wise correlations between rs2479106 and the other four SNPs are the same (measured as D'=95\%).

\section{Abbreviations}

BMI: Body Mass Index; DENNDIA: DENN Domain Containing 1A; E2: Estradiol; ERAP1,: Endoplasmic Reticulum Amino Acid Peptidase 1; FSH: FollicleStimulating Hormone; LD: Linkage Disequilibrium; LH: Luteinizing Hormone; LHCGR: Luteinizing Hormone/Choriogonadotropin Receptor; PCOS: Polycystic Ovary Syndrome; PRL: Prolactin; SNP: Single Nucleotide Polymorphism;

T: Total Testosterone; THADA: Thyroid Associated Protein

\section{Acknowledgements}

We are extremely grateful to all the women who agreed to participate in this study.

\section{Authors' contributions}

YW, WDY and QG conceived and designed the work; SMS, YXC and WJW were responsible for the recruitment of patient and control subjects and performed clinical examinations; YTZ, YNZ and QL performed the laboratory experiments; YNZ, YTZ, LY and YW analyzed the data; YNZ, YTZ and XZ wrote the manuscript. All authors read and approved the final manuscript.

\section{Funding}

The study was funded by the National Natural Science Foundation of China (81771539 \& 81971346), Nanjing Medical Science and Technique Development Foundation (ZKX17033 and YKK18127). The funding bodies played no role in the design of the study and collection, analysis, and interpretation of data and in writing the manuscript.

\section{Availability of data and materials}

The datasets generated and analysed during the current study are available in the NCBI BioSample \& BioProject database repository. The data are accessible via the accession number: SAMN13640133 \& PRJNA596822: human Phenotype or genotype (TaxID: 9606).

\section{Ethics approval and consent to participate}

This study was approved by the Ethics committee of Jiangsu Key Laboratory of Molecular Medicine, Medical School, Nanjing University. The patients and health women provided verbal informed consent and volunteered to participate in the study.

The ethics committee approved this form of consent. Every patient need the blood test when she came to the hospital. We just got $1 \mathrm{ml}$ blood sample while they were undergoing the clinical examinations and performed the genotype test. We don't give any additional interventions for the patients.

Consent for publication

Not applicable.

\section{Competing interests}

The authors declare that they have no competing interests.

\section{Author details}

'Department of Endodontics, Nanjing Stomatological Hospital, Medical School of Nanjing University, Nanjing, Jiangsu, China. ${ }^{2}$ State Key Laboratory of Analytical Chemistry for Life Science \& Jiangsu Key Laboratory of Molecular Medicine, Medical School, Nanjing University, Nanjing, Jiangsu, China. ${ }^{3}$ Divisions of Endocrinology, Drum Tower Hospital, Medical School of 
Nanjing University, Nanjing, Jiangsu, China. ${ }^{4}$ Department of Health Technology and Informatics, Faculty of Health and Social Sciences, The Hong Kong Polytechnic University, Hong Kong, China. ${ }^{5}$ Department of Obstetrics and Gynecology, Anhui Medical University, Hefei 230022, People's Republic of China. ${ }^{6}$ Centre of Reproduction, Department of Obstetrics and Gynecology, Memorial Hospital of Sun Yat-Sen University, Guangzhou 510120, People's Republic of China.

\section{Received: 11 May 2019 Accepted: 26 December 2019}

Published online: 15 January 2020

\section{References}

1. Chen J, Shen S, Tan Y, Xia D, Xia Y, Cao Y, Wang W, Wu X, Wang H, Yi L, et al. The correlation of aromatase activity and obesity in women with or without polycystic ovary syndrome. J Ovarian Res. 2015:8:11.

2. Azziz R, Woods KS, Reyna R, Key TJ, Knochenhauer ES, Yildiz BO. The prevalence and features of the polycystic ovary syndrome in an unselected population. J Clin Endocrinol Metab. 2004;89(6):2745-9.

3. Costello MF, Eden JA. A systematic review of the reproductive system effects of metformin in patients with polycystic ovary syndrome. Fertil Steril. 2003;79(1):1-13.

4. Prapas N, Karkanaki A, Prapas I, Kalogiannidis I, Katsikis I, Panidis D. Genetics of polycystic ovary syndrome. Hippokratia. 2009;13(4):216-23.

5. Chen ZJ, Zhao H, He L, Shi Y, Oin Y, Shi Y, Li Z, You L, Zhao J, Liu J, et al. Genome-wide association study identifies susceptibility loci for polycystic ovary syndrome on chromosome 2p16.3, 2p21 and 9q33.3. Nat Genet. 2011:43(1):55-9.

6. Shi Y, Zhao H, Shi Y, Cao Y, Yang D, Li Z, Zhang B, Liang X, Li T, Chen J, et al. Genome-wide association study identifies eight new risk loci for polycystic ovary syndrome. Nat Genet. 2012;44(9):1020-5.

7. Ke L, Che YN, Cao YX, Wu XK, Hu YL, Sun HX, Liang FJ, Sun J, Yi L, Wang Y. Polymorphisms of the HSD17B6 and HSD17B5 genes in Chinese women with polycystic ovary syndrome. J Women's Health (Larchmt). 2010;19(12):2227-32.

8. Day FR, Hinds DA, Tung JY, Stolk L, Styrkarsdottir U, Saxena R, Bjonnes A Broer L, Dunger DB, Halldorsson BV, et al. Causal mechanisms and balancing selection inferred from genetic associations with polycystic ovary syndrome. Nat Commun. 2015;6:8464.

9. Welt CK, Duran JM. Genetics of polycystic ovary syndrome. Semin Reprod Med. 2014;32(3):177-82.

10. Goodarzi MO, Jones MR, Li X, Chua AK, Garcia OA, Chen YD, Krauss RM, Rotter JI, Ankener W, Legro RS, et al. Replication of association of DENND1A and THADA variants with polycystic ovary syndrome in European cohorts. J Med Genet. 2012:49(2):90-5.

11. Zhang YJ, Li L, Wang ZJ, Zhang XJ, Zhao H, Zhao Y, Wang XT, Li CZ, Wan JP. Association study between variants in LHCGR DENNDIA and THADA with preeclampsia risk in Han Chinese populations. J Matern Fetal Neonatal Med. 2018:1-5.

12. Dallel M, Sarray S, Douma Z, Hachani F, Al-Ansari AK, Letaifa DB, Mahjoub T, Almawi WY. Differential association of DENND1A genetic variants with polycystic ovary syndrome in Tunisian but not Bahraini Arab women. Gene. 2018;647:79-84.

13. McAllister JM, Modi B, Miller BA, Biegler J, Bruggeman R, Legro RS, Strauss JF 3rd. Overexpression of a DENND1A isoform produces a polycystic ovary syndrome theca phenotype. Proc Natl Acad Sci U S A. 2014;111(15):E1519-27.

14. Cui L, Zhao H, Zhang B, Qu Z, Liu J, Liang X, Zhao X, Zhao J, Sun Y, Wang P, et al. Genotype-phenotype correlations of PCOS susceptibility SNPS identified by GWAS in a large cohort of Han Chinese women. Hum Reprod. 2013;28(2):538-44

15. Casarini L, Simoni M, Brigante $G$. Is polycystic ovary syndrome a sexual conflict? A review. Reprod Biomed Online. 2016;32(4):350-61.

16. Marat $\mathrm{AL}$, Dokainish $\mathrm{H}, \mathrm{McPherson} P S$. DENN domain proteins: regulators of Rab GTPases. J Biol Chem. 2011;286(16):13791-800

17. Allaire PD, Ritter B, Thomas S, Burman JL, Denisov AY, Legendre-Guillemin V, Harper SQ, Davidson BL, Gehring K, McPherson PS. Connecdenn, a novel DENN domain-containing protein of neuronal clathrin-coated vesicles functioning in synaptic vesicle endocytosis. J Neurosci. 2006:26(51):13202-12.

18. Allaire PD, Marat AL, Dall'Armi C, Di Paolo G, McPherson PS, Ritter B. The Connecdenn DENN domain: a GEF for Rab35 mediating cargo-specific exit from early endosomes. Mol Cell. 2010;37(3):370-82.

19. Olszanecka-Glinianowicz M, Banas M, Zahorska-Markiewicz B, Janowska J, Kocelak P, Madej P, Klimek K. Is the polycystic ovary syndrome associated with chronic inflammation per se? Eur J Obstet Gynecol Reprod Biol. 2007; 133(2):197-202.

20. Welt CK, Styrkarsdottir U, Ehrmann DA, Thorleifsson G, Arason G, Gudmundsson JA, Ober C, Rosenfield RL, Saxena R, Thorsteinsdottir U, et al. Variants in DENND1A are associated with polycystic ovary syndrome in women of European ancestry. J Clin Endocrinol Metab. 2012;97(7):E1342-7.

21. Eriksen MB, Brusgaard K, Andersen M, Tan Q, Altinok ML, Gaster M, Glintborg D. Association of polycystic ovary syndrome susceptibility single nucleotide polymorphism rs2479106 and PCOS in Caucasian patients with PCOS or hirsutism as referral diagnosis. Eur J Obstet Gynecol Reprod Biol. 2012:163(1):39-42.

22. Gao J, Xue JD, Li ZC, Zhou L, Chen C. The association of DENND1A gene polymorphisms and polycystic ovary syndrome risk: a systematic review and meta-analysis. Arch Gynecol Obstet. 2016;294(5):1073-80.

23. Gammoh E, Arekat MR, Saldhana FL, Madan S, Ebrahim BH, Almawi WY DENND1A gene variants in Bahraini Arab women with polycystic ovary syndrome. Gene. 2015;560(1):30-3.

24. Bao S, Cai JH, Yang SY, Ren Y, Feng T, Jin T, Li ZR. Association of DENND1A gene polymorphisms with polycystic ovary syndrome: a meta-analysis. J Clin Res Pediatr Endocrinol. 2016:8(2):135-43.

25. Lerchbaum E, Trummer O, Giuliani A, Gruber HJ, Pieber TR, ObermayerPietsch B. Susceptibility loci for polycystic ovary syndrome on chromosome 2 p16.3, 2p21, and 9q33.3 in a cohort of Caucasian women. Horm Metab Res. 2011:43(11):743-7.

26. Eriksen MB, Nielsen MF, Brusgaard K, Tan Q, Andersen MS, Glintborg D, Gaster M. Genetic alterations within the DENND1A gene in patients with polycystic ovary syndrome (PCOS). PLoS One. 2013;8(9):e77186.

27. Malini NA, Roy GK. Evaluation of different ranges of $\mathrm{LH}: \mathrm{FSH}$ ratios in polycystic ovarian syndrome (PCOS) - clinical based case control study. Gen Comp Endocrinol. 2018;260:51-7.

28. Chen L, Hu LM, Wang YF, Yang HY, Huang XY, Zhou W, Sun HX. Genomewide association study for SNPS associated with PCOS in human patients. Exp Ther Med. 2017:14(5):4896-900.

29. Ha L, Shi Y, Zhao J, Li T, Chen ZJ. Association study between polycystic ovarian syndrome and the susceptibility genes polymorphisms in Hui Chinese women. PLoS One. 2015;10(5):e0126505.

30. Danhong P, Luo J, Li L, Xuelin L, Mulan R. Meta-analysis on relationship between DENND1A polymorphisms and polycystic ovary syndrome. J Nanjing Med Univ. 2017:37(10):1362-8.

31. Saxena R, Georgopoulos NA, Braaten TJ, Bjonnes AC, Koika V, Panidis D, Welt CK. Han Chinese polycystic ovary syndrome risk variants in women of European ancestry: relationship to FSH levels and glucose tolerance. Hum Reprod. 2015;30(6):1454-9.

\section{Publisher's Note}

Springer Nature remains neutral with regard to jurisdictional claims in published maps and institutional affiliations.

Ready to submit your research? Choose BMC and benefit from:

- fast, convenient online submission

- thorough peer review by experienced researchers in your field

- rapid publication on acceptance

- support for research data, including large and complex data types

- gold Open Access which fosters wider collaboration and increased citations

- maximum visibility for your research: over $100 \mathrm{M}$ website views per year

At BMC, research is always in progress.

Learn more biomedcentral.com/submission 